This is the post print version of the article, which has been published in Information Technology and Systems:

Proceeding of ICITS 2019, p. 881-890.

https://doi.org/10.1007/978-3-030-11890-7_82

\title{
Minors and Artificial Intelligence - implications to media literacy
}

\author{
Jussi Okkonen, Sirkku Kotilainen \\ Tampere University, FIN-33014 Tampere University, Finland \\ \{jussi.okkonen, sirkku.kotilainen\}@uta.fi
}

\begin{abstract}
The artificial intelligent systems are reshaping human reality. Especially children and youth are altering their mobile communication and media usage fundamentally in their everyday lives today. During children's use the mobile artificial intelligent applications, the "robot" inside the application is learning from the feedback provided by children and youth through their uses. Based on review on current research and preliminary interviews, this paper addresses the topic from two perspectives. The first perspective is about acceptability of such technologies in general, i.e. to whom or what people are willing to grant right to control content, access and actions in digital sphere. The second perspective is about how users, and their parents too, are affected by the use of pervasive and immersive technology that shapes their media practices. The objective of the pilot study is to gain insightful knowledge of understandings of the artificial intelligence based adaptive media uses among children and youth together with practices of media and information literacies.
\end{abstract}

Keywords: artificial intelligence, minors, media literacy

\section{Introduction}

The artificial intelligent systems are reshaping human reality. They engulf our physical and mental bodies and they affect our everyday life and wellbeing via applications and services in general, but especially the wellbeing of minors. During children's use the mobile artificial intelligent (later AI) applications, the "robot" built in the application is learning from the feedback provided by children and youth through their uses. These personalized applications are based on the machine learning as the third generation AI (e.g.[1]). These applications learn from children's behaviour in their regular use. How about the user's trust and privacy? What kind of media and information literacies are needed?

User's trust in AI based applications and services is an ethical and moral question in the current information societies with changing digital environments. Therefore it is important to understand the construction of trust in children's practices with these digital media. For example, the European Parliament has pointed out these ethical questions related to the collection and using of personal data in global companies like Google and Microsoft. There always are new media with advertisers, content producers with their changing strategies to attract the consumers [2], as 
especially children and young more recently in new models of mobile devices having e.g. AI based applications like Bixby by Samsung corporation. This paper is discussing children's media use focusing on trust, privacy and personal information management together with media and information literacy from the perspective of youth agency (e.g. [3]) based on a pilot study with interviews among youth and their parents in Finland and South-Africa. Motivation for the pilot study is in developing a comparative research agenda.

There is some common conceptual core of trust to be enlightened. The definition of trust has studied in following domains: management, marketing, psychology, and sociology. Nevertheless, most authors working on trust provide their own definition, which is tailored for specific domain. [4] In this paper, we study children's trust in artificial intelligent applications. Artificial intelligent research settings the definition of trust needs to be re-formulated. In addition, there is little of empirical research in trust process of $\mathrm{AI}$ and children available.

The earlier research in artificial intelligent systems are written mostly in the technical point of view. [5] Research tradition among computer scientists is strong, only recently it has taken people also into focus. For example, in CHI conference, AI and user experience has started to bloom last few years (cf. www.chi.org). In educational sciences topic is also researched, still it mainly focus on how AI is taken as teaching assistant. For example, Serholt et al. [6] report the results of children's uncritical attitudes towards the teaching robot. Most probably, the minors' uncritical attitudes can been noticed in their everyday uses and practices of media as well.

The objective of the pilot study is to gain insightful knowledge of culturesensitive understandings of the artificial intelligence based adaptive media uses among children and youth together with practices of media and information literacies. The objectives turn into guiding research questions as such: 1) How young people are using adaptive media including AI from the perspectives of trust and privacy? 2) How young people are practising media and information literacies in their uses of adaptive media including AI? 3) How do the parents understand this new phenomenon in youngsters media use? acceptable.

\section{Minors, media literacy and trust}

Throughout the history of the research on children, youth and media the moral panics framework appear when new technologies and media is introduced to the markets. Kirsten Drotner [7] argues that each moral panic tends to follow an expectable route starting with pessimistic elitism associated with calls for technocratic and legalistic measures like censorship moving to optimistic pluralism calling for media literacies. Recent technological developments in media spheres have given space to integrative frameworks, which this study applies as well, respecting and increasing young people's agency from the perspective of the UN Convention of Rights of the Child to access, create, explore, express and learn media and information literacies [8]. The proposed study participates in current discussions of IT skills like coding as literacy [9] for recognizing algorithms and AI, as critical media literacies of today [10].

In this study we take a broad view of media literacy where 'media' refers to all the information technologies which provide public spaces and opportunities for 
interacting with people as audiences, and for participating in and through the media, embedded within social relationships. The term media includes the aspects of production, expression and reception as a process, which are also regarded as core elements of media literacies [11,12]. Media literacies has been defined in multiple ways and, more recently as MIL, i.e. media and information literacies, mostly promoted by UNESCO [13]. Media literacies as practices that help participation and engagement through and with the media include attitudes, critical judgments, and ethical reflections on the media among other skills [14]. Our hypothesis is, that these previous formulations of media literacies are not enough when we are dealing with artificial intelligence-based applications. Especially the new working logic of AI applications and services challenge the intellect of parents and minors.

Parental mediations strategies have been studied and defined, for example, in EUKIDS Online research (see, eukidsonline.net). Most probably, the previous suggestions of parents getting interested in children's cultures online or setting up family-based rules for using digital media still are valid, but need some updating.

This pilot study is based on the social constructionist perspective [15] according to which the society shapes technology and its use and, the empowerment and wellbeing of children and youth depends on various factors such as access to technological resources, motivations for internet use, policies and education as forming cultural and social capital for children and young.

In cognitive and social science, there is not yet a shared or prevailing, and clear and convincing notation of trust. Mutti [16] states that the number of meanings attributed to the idea of trust in social analysis is disconcerting. Certainly, this deplorable state of thing is the product of a general theoretical negligence [17]. For instance, social capital theory formed by James Samuel Coleman and Robert Putnam has two characteristic features in relation to trust: norms of reciprocity and trustworthiness.[18, 19]

The trust in guided decision-making is more and more depending on AI, whereas trust is built among children in different cultural backgrounds, variety of AI artefacts, media and commercial enterprises. Together they constitute network based platforms i.e. distributed global trust. Distributed trust is a paradigm shift driven by innovation technologies that rewriting the rules of an all-too-human relationship. We are already putting our faith in algorithms over humans in our daily lives, whether it is Amazon recommendations what to read or Netflix suggestions what to watch. In this context, more objective research and critical view in relation between AI and trust is needed.

The most relevant approaches to trust in the technological domain are: the logical approach, the computational approach and sosio-cognitive approach. In this paper, trust is implied as combination of Multi-Agent Systems (MAS) and sociocognitive approach. MAS prevails in the distribution of the various attitudes of agents who are possibly considering the emergence of the global phenomenon. and sosiocognitive approach, where trust imply the subjective probability of the successful performance given behaviour, and it is on the basis of the subjective perception/evaluation that the agent decides to rely or not, to bet or not on the trustee i.e. artificial intelligent artefact. [17]

This paper also take into account that trust is a relational construct. That involves a subject $\mathrm{X}$ as cognitive agent, an addressee $\mathrm{Y}$ (trustee) as an agent, which is 
capable of causing some effect as the outcome or its behaviour and causal process itself and its results, that is an act alfa of Y possibly production of desired outcome 0. [20] The global communication firm Edelman has been conducting an annual "Trust Barometer" asking more than 30000 people across twenty-eight countries on the level of trust. The media suffered the biggest blow, now distrusted in 82 per cent of all 28 countries surveyed. In UK, the number of people saying they trusted the media fell from 36 per cent in 2016 to 24 per cent in 2017. [21]

Social capital theory proposes that networks of relationships are a valuable resource for both individuals and organizations. According to Arenius [22] those networks are for interaction and for gaining access to assets required. Nahapiet \& Ghoshal [23] have observed that the value of the social capital is in its ability to make possible the achievements, which would be impossible without it or would be very costly, thus the value of social capital relies on its usability. Social capital is invisible, ubiquitous, and hard to pindown, so it is usually studied in terms of its manifestations and effect[23]. Social capital can operate at the level of an individual, a team, an organization, an industry, a community, a nation, or an entire economy (e.g. Putnam 1993). According to Arenius [22] the social capital theory suggests that players are able to gain access to various kinds of resources that accrue to them by virtue of their engagement in various kinds of relationships. Resources are available through the contacts or the connections that networks bring. As well as conveying resources, social capital is a means of enforcing norms of behavior among individuals or corporate actors, and thus acts as a constraint.

In inter-personal relationships, or in this case hybrid relationships, social capital facilitates learning because offers access to knowledge and enables knowledge transfer [23]. More social capital, i.e. interaction between different actors, trust and mutual understanding, more efficient is the process of transferring explicit, and especially tacit knowledge. Moreover, it promotes acceptance of information provided.

In the context of using applications and services social capital should be taken in account as sum of more or less institutionalised relationships of mutual acquaintance and recognition or network of social exchanges between operatives or actors engaging in transactions (cf. e.g.[24, 25, 26]). Moreover the three dimensions of social capital affecting any actor in that process are structural, i.e. presence or absence of interaction, dimensional, i.e. mutual trust and trustworthiness, and cognitive, i.e. shared understanding of common goals and proper ways to act [27, 28, 29]. Structural dimension of social capital is often emphasized when considered relationships of mutual acquaintance that forms a basis for activities. From the perspective of user the gains are twofold as recognition and knowing different participants operative actions that structural social capital offers access. Without relationships of acquaintance the involvement cannot be fulfilled. One might even claim that structural social capital is the source of the first move in any activity, since at least on party of interaction should acknowledge counterparners.

Dimensional dimension of social capital is most important in early trust building. Participant must be sure that any information she or he submits to will be valued. From the perspective of single user dimensional social capital is the issue of trustworthiness. It is the issue of commitment to the decided goal or avenue of action. On the other hand user is interested in commitment of the others, as the network based 
on structural social capital cannot hold if there is commitment to other goals than the (explicitly) agreed.

Cognitive dimension of social capital consist of the social exchanges between actors engaging in the process. It is the issue of understanding on "where we are now, and where we are going, what is desirable". Cognitive dimension is shared understanding on what is the primary goal. It is recognition of meaning or rationale for their interaction. From the perspective of user cognitive dimension is the recognition of the goal for activity and knowledge of legitimate ways to achieve the goal. It is more or less issue of what can and should be done. Moreover, it is also knowledge on who to know, i.e. how the network is build. The cognitive dimension can be considered supportive to other two dimensions, as it contains the shared norms. Having knowledge on how one can and should behave in certain situation is important, because without that knowledge networks can be ruined and trust lost.

As trust is built in interaction the social capital theory is most relevant in sense, that the presented dimensions seem to exist in using adaptive platforms or services. The interaction with such technology is of course more straightforward than in more complex social situations, yet analogies are found. Structural dimension is about acknowledging technology as a counterpart, yet doing so user also recognizes mutual relationship to build trust on. Dimensional social capital in this context is issue of trust, i.e. gaining of technology by added value or pure enjoyment. Cognitive dimension is one sided, as by the trust build people tend to act according to technological determinism. Moreover, such assumption on technology side implicit, since it react on previous queries or actions. The same dimension could be found in interaction with technology as in social interaction. This is an important notion when researching how people use and adapt to digital environments optimized by algorithms.

\section{Issues of artificial intelligence}

In order to better understand the phenomenon a series preliminary interviews were conducted with 16 pair of a minor and a parent with 10-17 years aged children in Finland (9 pairs) and South Africa ( 7 pairs). The pairs were recruited in casual situations in airport based on observation of using of mobile device. The selection of interviewees was based on convenience sampling and voluntary cooperation. In addition, 5 pairs refused to be interviewed. The unstructured interviews shed light on the issue in general and served as initial data on the topic. People were asked if they could share their opinion on using their device. The interviews had two main questions. One aimed for the minor: "What do you think about artificial intelligence in your device and the services you use?" The second was aimed for the parent: "What do you think about the artificial intelligence in the device and the services your child is using?". The interviews were conducted in free form discussions about 10 to 15 minutes. The aim for such brief interviews was to map attitudes on the topic. Moreover, aim was to build understanding on how people see the role of technology in the devices and services. Even the sampling is biased and the results are just scratching the surface on the topic several relevant notions could be found.

The interviews addressed the topic from two perspectives. The first perspective is about acceptability of such technologies in general, i.e. to whom or what minors and parents are willing to grant right to control content, access and actions in 
digital sphere. The second perspective is about how users are affected by the use of pervasive and immersive technology that shapes their media practices. This is an issue of media literacy, as initial interviews revealed the minors or parent showed to have no understanding or they were indifferent on how AI shapes the digital landscape.

The minors showed no awareness on technology issues or even situations when they are exposed to AI. Not a single interviewed minor had idea of the technology optimizing content for services such as Facebook, Instagram, Snapchat, Youtube. For them it was ok to grant access to their actions in digital environment and they found service providers trustworthy in sense they granted access to their credentials, locations, contents, contacts for example. Moreover, recommendations by applications and services were welcomed and taken as good service rather than side effect of surrendering their privacy.

$$
\begin{aligned}
& \text { “I don't mind."[MF16] } \\
& \text { “AI helps you." [MSA1] }
\end{aligned}
$$

"I mostly use my phone for discussion and sharing photos and videos. I like when I get notifications what my friends have posted." [MF2]

"It is nice to know what my circles are doing."[MSA2]

AI, i.e. algorithms, increases attractiveness and immersiveness of applications or services according to minors due to better user experience. Most of them told that they appreciate content that suits their taste and habits. Advertisements are easy to skip, so they do not mind to be exposed to those. 7 out of 16 told that they are sometimes annoyed due to social media content that is offered to them. In most services, content offered to them was found interesting and therefore it increased attractiveness of such service. Most of them also were active to browse recommendations as a source of new vistas.

"You can spend long time in Youtube watching really interesting content e.g. on your hobbies or fashion." [MSA3]

"The best thing is when you google something and you have lot of content." [MF15]

Parents did not pay much attention AI features of applications or services the minors use. They were more aware of such elements in services and applications, yet they trusted service and content provider to honor age limits and privacy. Most of the parents openly told that they are not interested or have no understanding on issue. Parents were more concerned actions taken by other online users, and to some extent commercial motives of corporations while exposing minors to well targeted marketing efforts

"I'm very skeptical on service algorithms, yet I trust google that Iphone knows my child's age and content is suitable." [PF4] 
"The phone is somewhat like an extension of life for my child. He seems to be happy with it. I'm concerned on the privacy, not on the commercial content." [PF9]

“Actually I don't care how digital environments work since they are relatively safe for kids." [PSA9]

"I'm not an engineer; easy use devices makes life much easier." [PF11]

Acceptability of AI, i.e. trust of AI, could be summarized on these findings. Children can make distinction between paid and other content, yet they cannot evaluate the difference on validity and relevance of it to them. Manipulation of the news feeds, search results, etc. was found irritating, yet most of them thought it is impossible to escape it. AI is accepted, even appreciated, as it provides content and information related to previous interest.

"Most [search] results seem useful." [MF4]

"I don't mind if there is advertisements." [MSA7]

"Sometimes, or quite often actually, you are recommended to watch videos of totally random people. If you are in hurry it is very annoying." [MF12]

AI is accepted or tolerated since parents see no way to bypass it. They commented that ever since the google and cookies all actions in digital environment are subject to intelligence and manipulation. Avoiding such technology was found too troublesome. Parents thought their children are not "google"-litterate and it concerns them to some extent, since minors are not critical to content they see.

“It [algorithms] is everywhere." [PF10]

"I think people are very happy if there is quick way to find content on their interests." [PSA4]

"School is [nowadays] about using internet to acquire information for assignments. It is convenient for them [children].” [PF2]

Media practices were issue by the parents since AI changes media practices. Several parents brought about their concern because the content is filtered and optimized according to user. Parents also discussed that AI blocks serendipity, because of it is tempting to grasp the "first" option. They thought that critical attitude towards the content requires effort and minors are a bit lazy. One parent stated that it does not require or leverage critical reading skills, as the most "'relevant' is on the top". Parents also claimed that children cannot make clear distinction between optimized content, 
e.g. advertisements, and other content. In general AI challenges media literacy, since $\mathrm{AI}$ awareness of minors is low, even non existing.

"I'm concerned on how children take the first option and follow the path on Youtube or Google. At least mine [child] does not have a clue on critical media skills." [PF2]

\section{Discussion and conclusions}

The first key finding was that minors are indifferent on AI, but it concerns parents. The awareness of AI is low and the effects are ambiguous. Moreover, both minors and parents have no comprehension of AI. It is understood through popular culture artefacts such as science fiction novels for example. The second key finding was that minors trust AI as it acknowledged as a smart helping agent. Parents are somewhat concerned on that trust. The third key finding is that parents thought the minors are not AI literate and attention to it should be paid more in the future as algorithms shape more digital sphere.

The AI and minors is topic that not even existed earlier, therefore there is a need for reformulation of media literacies in the digital societies. Based on this pilot study, it seems that further discussion and integration of media literacies and information literacies, even coding as literacies are needed cf. [30, 9]. Media and information literacies belongs to the core civic skills of current digital societies which rely more and more on the discursive power making through communication. As such, for example, the spreading of disinformation aims to guide thinking and actions of the user with hiding some facts, not telling the whole truth like in so called fake news or in hate speech which is about hateful, discriminative expressions of minorities like ethnic groups in societies. Users understanding of the methods of algorithms and artificial intelligence belong to critical media and information literacies (MIL) of today.

How do children build the trust to AI artefacts? During the play AI artefacts behavioural model is becoming gradually familiar and children are capable to recognise the familiar behavioural models. The problematic is when children start to trust familiar behavioural models and advices of AI artefacts, which might not be trustworthy. It is evident, that children can form a trust to AI artefacts very fast. However, how long it takes that they learn to mistrust them, if the artificial intelligent artefacts betray them in any circumstance, it is expected that distrust appear.

This initial study on the topic opened a new research avenue on human technology interaction and media literacy. The findings support the proposition that new technology enables attractive and easy to use applications and services, yet they change how people use information and media. Based on insights of the interviewed people a straightforward conclusion is that they are happy with user-friendly technology. This somewhat rough conclusions on limited data leads to new research challenges. At the end, children needs to understand trust and AI in cognitive level. They need to understand that AI dynamic algorithms are based on their own actions. And the most important, they need to understand that control and power of media and interaction is in their own hands. Need for media literacy 3.0 is essential. The unique real answer for coping with others' autonomy is to establish a real trust relationship. Finally, the ability to understand and model the trust concept to transfer its utility in the 
technological co-operation framework, will be in fact the bottleneck of the development of the artificial intelligent that is technology of the future.

One of the key questions then is how can the educational system and educational policy answer to the need of conceptual and pedagogical development? This study is calling for further research together with reformulations of national, paneuropean and global educational policies. Moreover, there is a need for a research agenda for contemplating the multi-faceted phenomenon of artificial intelligence and minors.

\section{References}

1. Schmidhuber, J. (2015) "Deep learning in neural networks: An overview." Neural Networks 61, 85-117.

2. van Dijk, J. (2009) Users like you? Theorizing agency in user-generated content, Media, Culture and Society, Volume 31, Issue 1, 41-58. DOI: 10.1177/0163443708098245

3. Frau-Meigs, D, Flores, J. \& Velez, I. (2017) Public policies in media and information literacy in Europe: cross-country comparisons. London: Routledge.

4. Castelfranchi, C. \& Falcone, R. 2010. Trust Theory. A Socio-Cognitive and Computional Model. Wiley Series in Agent Technology.

5. Aly, A., Griffits, S., Stramandinoli, F. (2017) Towards intelligent social robots: Current advances. Cognitive robotics.

6. Serholt, S. et. Al. (2017) "The case of classroom robots: teacher's deliberations on the ethical tensions." Artificial Intelligence \& Society 4(32)

7. Drotner, K. (1992) 'Modernity and media panics', in M. Skovmand \& K.C. Schroeder (eds.)

Media Cultures: reapprising transnational media, 42-62. London: Routledge

8. Livingstone, S. (2014) 'Children's digital rights: a priority'. Intermedia, 42:4/5, 20-24.

9. Dufva, T. \& Dufva, M. (2016) Metaphors of code - Structuring and Broadening the discussion on teaching children to code. Thinking Skills and Creativity 22 (2016), DOI: 97110.10.1016/j.tsc.2016.09.004

10. Kotilainen, S. \& Ruokamo, H. (2017) 'Opettajankoulutus Haloo: TVT ja medialukutaitojen opetus yhteen!' [Hello Teacher Education: ICT and Media Literacies belong together!] In Salomaa, S., Palsa, L. \& Malinen, V. (2017) Opettajaopiskelijat ja mediakasvatus. Kansallisen Audiovisuaalisen Instituutin julkaisuja 1/2017.

11. Buckingham 2003

12. Jenkins et al. 2009

13. Frau-Meigs, D, Flores, J. \& Velez, I. (2017) Public policies in media and information literacy in Europe: cross-country comparisons. London: Routledge.

14. Livingstone, Sonia (2008) Engaging with media - a matter of literacy? Communication, culture \& critique, 1 (1). pp. 51-62. DOI: $10.1111 /$ j.1753-9137.2007.00006.x

15. Berger, Peter L. \& Luckmann, T. (1966) The social construction of reality: A treatise in the sociology of knowledge

16. Mutti, A. 1987. La fiducia. Un concetto fragile, una solida realta, in Rassegna italiana de sociologia, pp. 223-247.

Putman, R. 1995. Bowling Alone: The Collapse and Revival of American Community. Journal of Democracy. 6 (1): 65-7

17. Castelfranchi, C. \& Falcone, R. 2010. Trust Theory. A Socio-Cognitive and Computional Model. Wiley Series in Agent Technology. 
18. Putnam RD. Tuning In, Tuning Out: The Strange Disappearance of Social Capital in America. P.S.: Political Science and Politics [Internet]. 1995;28 (4) :1-20

19. Putman, R. 2000. Bowling Alone: America's Declining Social Capital. Culture and Politics. Springer Link. pp. $223-234$.

20. Castelfranchi, C. Towards an agent ontology: autonomy, delegation, adaptivity. AI'IA Notizie. 11 (3): 45 - 50, 1998: Special Issue on "Autonomous Intelligent Agents", Italian Association for Artificial Intelligence, Roma

21. Botsman, R. 2017. Who can you trust? How technology Brought Us Together and Why It Might Drive Us Apart. Hachette Book Croup

22. Arenius, Pia (2002), Creation of Firm Level Social Capital, Its Exploitation, and Process of Early Internationalization. HUT Dissertations 2002/3, Espoo.

23. Naphiet, J \& Ghosal, S. (1998), Social Capital, Intellectual Capital and the Organisational Advantage. Academy of Management Review 22(2): 242-266.

24. Seppä and Näsi 2001,

25. Sorheim, R. (2003), The pre-investment behaviour of business angels - A social capital approach. Euram 2003 3.-5.4.2003 Milan, Italy

26. Davidsson, P., Honig, B. (2002), The role of social and human capital among nascent entrepreneurs. Journal of Business Venturing 5210 (2002). Elsevier Science Inc

27. Yli-Renko, H. (1999), Dependence, Social Capital, and Learning in Key Customer Relationships: Effects on the Performance of Technology-Based New Firms. Acta Polytechnica Scandinavica, Espoo.

28. Bourdieu, P and Waquant, L.J.D. (1992), Invitation to Reflexive Sociology. The University of Chicago Press.

29. Ilmonen, K. (ed.) (2000), Sosiaalinen pääoma ja luottamus. SoPhi, Jyväskylä.

30. Pathak-Shelat, M. \& Kotilainen, S. (2015) "Media and Information Literacies and the Wellbeing of Young People: Comparative perspectives" in Kotilainen, S. \& Kupiainen, R. (eds.) Reflections on Media Education Futures: Contributions to the Conference Media Education Futures in Tampere, Finland 2014. Gothenburg: Nordicom, 147-158. 